\title{
Spazmolitik Etki Gösteren Bazı Flavanon Eter Türevleri Üzerinde Sentez Çalışmaları II *
}

Essais de Synthese sur Quelques Derives d'Ether de Flavanone ayant l'Activite Spasmolytique II.

\section{Rahmiye ERTAN **}

\section{GíRiş}

Khellin adi verilen 2 - metil - 5,8 - dimetoksi - 6,7 - (2'3' - furano) kromon yapısındaki bileşiğin spazmolitik etkisinin kromon halkasından ileri gelebileceği düşüncesinden hareketle "Flavon" (= 2fenil kromon) bileşikleri üzerindeki sentez çalışmaları sırasında "Efloxate, Recordil" isimleri ile tanınan, 7 - Flavonoksi - asetik asit etil esterine ulaşılmıştır (1). Bundan önceki çalışmamızda bu bişikteki flavon halkası yerine "Flavanon" halkası ele alınıp 7. konumda oluşturulan serbest $-\mathrm{OH}$ grubu üzerinden, $\alpha$ - bromo - $\alpha$ sübstitüe asetik asit etil esterlerinin eter türevleri sentezlenmiş ve elde edilen ürünlerin Efloxate referans alınarak, asetükolin, $\mathrm{BaCl}_{2}$ ve histamin agonistlerine karş1 spazmolitik etkileri incelenmiştir (2). Bu incelemeler sonucu bazı türevlerin her üç agoniste karş1 \% 80 nin üstünde bir inhibisyon göstermeleri ve düz kaslar üzerine tersinir bir etki içermeleri bu kez 7. konumdaki grubun sabit tutularak B halkasından yapılacak sübstitüsyonun aynı aktivitedeki etkisini araştırmayı düşündürmüştür. Böylece $\mathrm{B}$ halkasından, p-metoksi grubu içeren türevler sentezlenerek spazmolitik açıdan incelenmeleri amaçlanmıştır.

\section{MATERYAL ve METOD}

Elde edilmesi düşünülen bileşiklerin sentezleri için gerekli maddelerden asetonitril (BDH), rezorsinol (Merck), p-anizaldehit (Merck), $\alpha$ - bromoasetik asit etil esteri (BDH), $\alpha$ - bromobütirik asit etil esteri (Merck - Schuchardt), $\alpha$ - bromovalerik asit etil es-

Redaksiyona verildiği tarih : 26 Kasım 1981

* Aynı adlı Doçentlik tezinin (1979) bir bölümüdür.

** Farmasotik Kimya Kürsüsü, Eczacılık Fakültesi, Ankara Üniversitesi. 
Spazmolitik Etki Gösteren Bazı Flavanon Eter Türevleri

teri (Merck - Schuchardt) ve temin edilemeyen rezasetofenon. $\alpha$ - bromopropiyonik asit etil esteri, $\alpha$ - bromofenil asetik asit etil esteri de tarafımızdan sentezlendi. Ayrıca referans bileşik olarak kullanılan Efloxate, literatürde verilen patent yöntemi ile (1) elde edildi.

Bu çalışmada da (I). bölümde olduğu gibi, sentezlenen bileşikleri 2 ayrı bölümde ele almak gerekmiştir :

\section{$1-7$ - Hidroksi - 4' - metoksi flavanon $\left(=\mathbf{F}_{2}\right)$ sentezi :}

a) Rezasetofenon sentezi : Bundan önceki çalışmada verilmiştir (2).

b) Şalkon sentezi : 2'4' - Dihidroksi - 4 - metoksi Şalkon $\left(=\mathrm{F}_{2} \mathrm{~S}_{\mathrm{S}}\right)$. Bundan önceki çalışmada tartışılan yöntem uygulanmış ve \% 75 verimle istenen maddeye ulaşılmıştır. Verimdeki bu yükseliş ilgi çekici olup oluşan maddelerin molekül yapıları incelenerek şöyle açıklanabilir (Formül I) :

$F_{1} S ̧$ türevinde (=2'4' - Dihidroksi Şalkon) karbonil fonksiyonu, rezasetofenon karbonilinden farklı olarak etilenik çift bağ aracılığı ile $\mathrm{B}$ halkası ile de rezonansa girecektir. Bu etkileşme $\mathrm{F}_{2} \mathrm{~S}_{\mathrm{S}}$ de $\mathrm{B}$ halkasındaki p-metoksi grubunun $+\mathrm{M}$ etkisi ile daha da güçlenecek ve sonuç madde olan şalkon daha kararlı bir yapıda olacağı için, reaksiyon termodinamik kontrol altında şalkon verecek şekilde yönlenecek ve verim yükselmiş olacaktır.

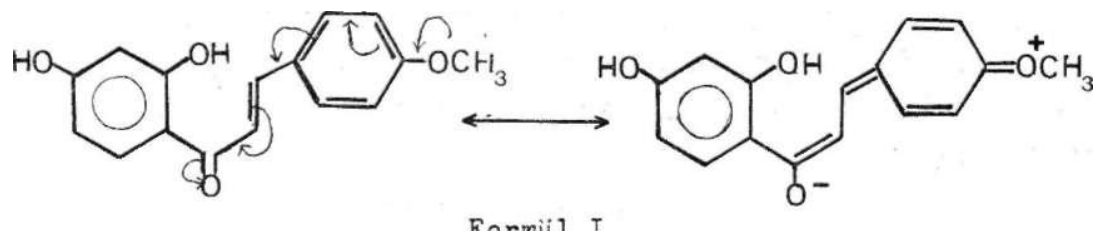

Formiil I.

c) Flavanon izomeri zasyonu : I. Bölümde geliştirilen yöntemle sağland1.

2 - 7 - Hidroksi - 4' - Metoksi Flavanon'un $\alpha$ - Sübstitüe $\alpha$ - bromo asetik asit etil esteri ile eter türevlerinin sentezi : $\mathbf{F}_{1}$ de olduğu gibi $F_{2}$ de de 7 . konumda bulunan serbest -OH fonksiyonu üzerinden, Efloxate örneğinde olduğu gibi, bir ester grubu taşıyan yan zincir eter köprüsü ile halkaya bağlanabilecektir. Bu konum- 


\section{Rahmiye ERTAN}

dan halkaya bağlanacak yan zincirlerin sabit tutulması düşünüldügü için $F \imath$ türevlerinde gerçekleştirilen bütün işlemler burada da aynen sağlanmıştır. Uygulanan reaksiyonlar sırasında $F_{1}$ e oranla büyük bir farklılık gözlenmemiştir. Sonuçta aşağıdaki türevler elde edilmiştir (Formül II) :

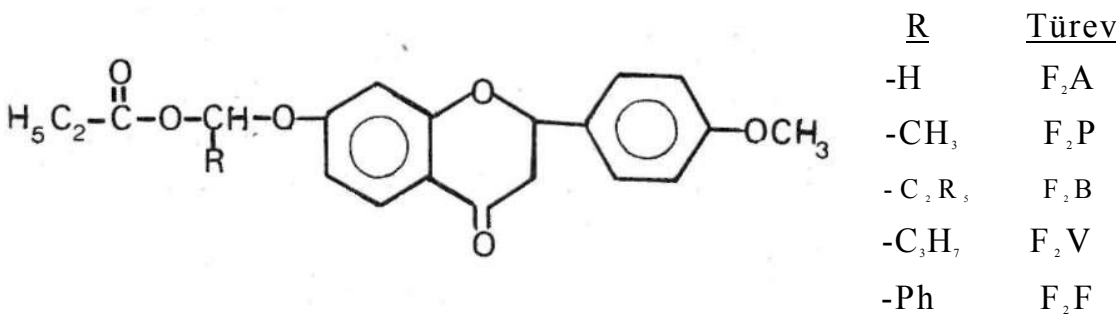

Formül II .

Ayrıca, yine $F_{1}$ örneğinde olduğu gibi $F_{2}$ halkasının da 7 - metoksi türevi $\left(\mathrm{F}_{2} \mathrm{M}\right)$ hazırlanmış ve diğer ester-eter yan zincirli türevlerle aktivite açısından mukayese edilmiştir.

\section{DENEYSEL BÖLÜM}

Gerekli kromatografik çalışmalarda $0.250 \mathrm{~mm}$ adsorban kaplı plaklar kullanıld1. Yararlanılan adsorban ve solvan sistemleri :

Adsorban: Kieselgel G (Merck) + Kieselgel $\mathrm{HF}_{254}$ (50:50)

Solvan sistemleri: A) n-Heksan + Etil asetat (80:20)

B) n-Heksan + Kloroform $90: 30)$

C) $\mathrm{n}$-Heksan + Aseton (60:40)

D) Petrol eteri $($ Kn. 35-60 $)+$

Aseton

Lekelerin belirlenmesinde UV lambası ( $254 \mathrm{~nm}$ dalga boyu) ve $\% 5 \mathrm{FeCl}_{3}$ (Metanollü), \% $15 \quad \mathrm{H}_{2} \mathrm{SO}_{4} /$ Metanol reaktifterinden yararlanild1. di.

En. tayinleri Büchi SMP - 20 aletinde kapiller yöntemle yapıl-

Elemanter analizler mikrometodla, UV Spektral ölçmeleri PYE-UNICAM SP -1700, IR spektrumları PYE - UNICAM SP - 1100 spektrofotometresinde $\mathrm{KBr}$ diski hazırlanarak alındı. NMR Spektrumları VARIAN - T - 60 ve Mass spektrumları EAI - Ms - 30 spektrometresinde elektron iyonizasyon yöntemi ile yapıldı. 


\section{Spazmolitik Etki Gösteren Bazı Flavanon Eter Türevleri 2'4' - Dihidroksi - 4 - Metoksi Şalkon (= 2.4 - Dihidroksifearil - 4 - Metoksi Stiril Keton) $=\mathbf{F}_{2} \mathbf{S}$ :}

$7.60 \mathrm{~g}(0.05 \mathrm{~mol})$ rezasetofenon, $40 \mathrm{mi}$ aldehitsiz etanolde çözüldü, üzerine $6.8 \mathrm{~g}(0.05 \mathrm{~mol})$ anizaldehit katıldı ve $38 \mathrm{~g} \mathrm{KOH'ın}$ $38 \mathrm{ml}$ sudaki yeni hazırlanıp soğutulmuş çözeltisi, oda sıcaklığında bir magnetik karıştırıcı ile karıştırılarak yavaş yavaş damlatıldı. Elde edilen koyu kahverenkli çözelti $60^{\circ} \mathrm{C}$ lik sıcaklıkta 2 saat tutuldu ve sonra oda sicaklığında 1 gece bekletildi. Buzlu su ile 1/2 oranında seyrettildi ve $50 \mathrm{ml}$ eter ile 2 kez tüketilerek reaksiyona girmeyen benzaldehit uzaklaştırıldı. Sulu faz buz banyosunda soğutularak, $\mathrm{PH}=4-5$ oluncaya kadar $6 \mathrm{~N} \mathrm{HC1}$ ile asitlendirildi. Böylece oluşan yağımsı çökelek buz dolabında 1 gece bekletilerek katılaşması sağlandı, sonra nuçeden süzüldü. $60-70^{\circ} \mathrm{C}$ lik su ile yıkanarak rezasetofenondan kurtarıldı. Asetonda çözülüp, aktif kömürden geçirildi. Aseton-metanolden kristallendirilerek saflaştırılan $10,1 \mathrm{~g}$ turuncu renkli bir madde elde edildi. Verim \% 74.8, En. $184-188^{\circ} \mathrm{C}\left(186^{\circ} \mathrm{C} \mathrm{Lit}\right)$ (3). $\% 5 \mathrm{FeCl}_{3}$ ile morumsu kahverenk, $\mathrm{NaOH}$ ile turuncu - kirmızı renk verir. $\mathrm{Rf}-0.57$ (solvan $\mathrm{C}$ ), 0.70 (solvan D).

$\mathrm{UV} \lambda_{\max }($ metanol $): 208 \mathrm{~nm}(\log \varepsilon=4.29), 238 \mathrm{~nm}(\log \varepsilon=$ $0.04), 364 \mathrm{~nm}(\log \varepsilon=4.57)$. IR. $(\mu): 3.09$ (yaygin $-\mathrm{OH}$ band1), $5.17(\mathrm{C}=\mathrm{O}), 6.32$ ve $6.71(\mathrm{C}-\mathrm{C}$ halka titreşim $), 9.2) 1-9.43$ ve 12.19 (1.4 disübstitüe oramatik halka deformasyon) (4), 7/84-8.33 (asimerik alkil - artil eter), 10.52 - 10.10 (trans şalkon) $(5,6,7)$. NME (İnt. TMS, $\mathrm{CDCl}_{3}, \delta \mathrm{ppm}$ ) : 7.8 (d. A halkasının 6' $-\mathrm{H}$ ), 6.4 (q. 5' -H), 6.3 (d. 3' -H), 6.8 (d. B halkasinin 3,5 - protonlar1), 7.6 (d. 2,6 - protonlar1). Etilenik grubun protonlar1 ise 7.6 - 7.8 de halka protonları ile örtülmüş olarak izlenmekte, 3.8 (s. $3 \mathrm{H}$ integrasyon değeri ile 4' - metoksil), 13.55 (2' -OH), 10.1 (4' -OH). Anal. : $\mathrm{C}_{16} \mathrm{H}_{14} \mathrm{O}$, için; Hesaplanan : C : 71.11; H : 5.18, Bulunan $\mathrm{C}: 70.98$; $\mathrm{H}: 5.02$.

\section{7 - Hidroksi - 4' - Metoksi - Flavanon $=\mathbf{F}_{2}$}

$4 \mathrm{~g}(0.015 \mathrm{~mol}) \mathbf{F}_{2} \mathbf{S}$ üzerine $20 \mathrm{ml}$ etanol, $60 \mathrm{ml} \% 1.5 \mathrm{NaOH}$ çözeltisi ve $20 \mathrm{~g}$ sodyum asetat $\left(\mathrm{CH}_{3} \mathrm{COONa} ; 3 \mathrm{H}_{2} 0\right)$ ilave edildi, $100^{\circ} \mathrm{C}$ lik yă banyosunda 2 saat geriçeviren soğutucu altında kaynatıldı. Bir gece oda sıcaklığında bekletildi, $80 \mathrm{ml}$ su ilavesiyle seyreltildi ve \% 20 lik asetik asit ile asitlendirildi, $\mathrm{pH}=7$ civarında çö- 


\section{Rahmiye ERTAN}

kelme başladi. Asitlendirmeye $\mathrm{pH}=5-6$ oluncaya kadar devam edildi, 2 saat buz dolabında bekletildikten sonra nuçeden süzüldü, su ile y1kanarak kurutuldu. Ham flavanon benzen ile muamele edilerek İçerdiği şalkonundan büyük ölçüde temizlendi. Metanolde çözülen ham flavanon sicakta aktif kömürden geçirildi. Aseton-metanol karışımından elde edilen kristallerin En : $183-185^{\circ} \mathrm{C}\left(184.5-185^{\circ} \mathrm{C}\right.$ (Lit. 8)), verim \% 51.

\% $5 \mathrm{FeCl}_{3}$ ile renksiz, $\mathrm{NaOH}$ ile sar1. Shinoda testi ile mor-menekşe renk gözlendi. Rf $=0.25$ (solvan A), 0.54 (solvan $\mathrm{C}$ ).

$\mathrm{UV} \lambda_{\max }($ metanol $): 216 \mathrm{~nm}(\log \varepsilon=4.45), 276 \mathrm{~nm}(\log \varepsilon=$ 4.27) ve $314 \mathrm{~nm} \quad(\log \varepsilon=3.95)$. IR. $\quad(\mu): 3.09(7-\mathrm{OH}), 6.09$ $(\mathrm{C}=\mathrm{O}), 6.36-6.71$ ve $6.92(\mathrm{C}-\mathrm{C}$ halka titreşim $), 7.84-8.33$ (aril - alkil asimetrik eter deformasyon), 12,04 (B halkası protonlarının düzlem dışı deformasyon vibrasyon). NMR. (İnt. TMS, $\mathrm{CDCl}_{3}, \delta \mathrm{ppm}$ ) : 8.00 (d. H - 5, 7 -OH ile birlikte), 7.7 (d. 2', 6' - protonlar1), 7.2 (d. 3', 5'- protonlar1), 6.6 - 6.8 (q. H-6), 6.6 (d. H- 8 $\mathrm{H}-6$ protonu ile birlikte), 5.5 (q. $\mathrm{H}-2 ; \mathrm{J}_{\text {trans }}=11 \mathrm{~Hz}, \mathrm{~J}_{\text {cis }}=5$ $\mathrm{Hz}$ ), 3.9 (s. 4' $-\mathrm{OCH}_{3}$ ), 2,9 (d. $\mathrm{H}-3_{\text {ax }} ; \mathrm{J}=11 \mathrm{~Hz}$ ), 2,8 (d. d. $\mathrm{H}-3_{\text {。q }}$ $\mathrm{J}=5 \mathrm{~Hz})$. Mass. : $\mathrm{m} / \mathrm{e}=270\left(\mathrm{M}^{+}\right), 269,163,137,134,119,108$, 91, 77. Anal. : $\mathrm{C}_{16} \mathrm{H}_{14} \mathrm{O}_{4}$ için Hesaplanan : $\mathrm{C}: 71.11 ; \mathrm{H}: 5.18, \mathrm{Bu}-$ lunan : $\mathrm{C}: 70.90 ; \mathrm{H}: 5.18$.

114 - Okso - 2 - (4' - metoksifenil) - 2,3 - dihidrobenzopiran - 7 - ill oksil asetik asit etil esteri; 7 - (4'-metoksiflavanon) oksi asetik asit etil esteri $=\mathrm{F}_{2} \mathrm{~A}: 0.405 \mathrm{~g}(0.0015 \mathrm{~mol}) \mathrm{F}_{2}$ üzerine $0.250 \mathrm{~g}$ $(0.0015 \mathrm{~mol}) \alpha$ - bromo asetik asit etil esteri, $0.207 \mathrm{~g}(0.0015 \mathrm{~mol})$ susuz $\mathrm{K}_{2} \mathrm{CO}_{3}$ ve $25 \mathrm{ml}$ kuru aseton ilave edildi. Karışım, bir magnetik karıştırıcı ile karıştırılarak, $\mathrm{CaCl}_{2}$ kurutma başlığ çeviren soğutucu altında 10 saat kaynatıldı. Aseton distillendikten sonra ortama $30 \mathrm{ml}$ su ilave edildiğinde hemen yağlımsı bir bölüm ayrıldı. Bu karışım $3 \mathrm{kez} 50 \mathrm{ml}$ eter ile tüketildi. Birleştirilen eterli ekstreler 2 kez $20 \mathrm{ml} \% 10$ luk $\mathrm{NaOH}$ ile tüketildi. Yıkama suyu nötr oluncaya kadar su ile yıkandı. Eterli çözelti susuz $\mathrm{Na}_{2} \mathrm{SO} 4$ üzerinden kurutuldu, aktif kömürden geçirildi, renksiz eterli çözelti yoğunlaştırıldı. Petrol eteri ilavesiyle çöktürülen madde eter-etanol karışımından kristallendirildi, verim : \% 73. En. : $101-102^{\circ} \mathrm{C}$. Eter ve asetonda çok çözünür, $\mathrm{MeOH}, \mathrm{EtOH}, \mathrm{CHCl}_{3}$ vc petrol ete- 


\section{Spazmolitik Etki Gösteren Bazı Plavanon Eter Türevleri}

rinde çözünür. \% $5 \mathrm{FeCl}_{3}$ ile renksiz, Shinoda testi ile mor-menekşe renk verir. $\mathrm{Rf}=0.18$ (solvan $\mathrm{A}), 0.64$ (solvan $\mathrm{C}$ ).

$\mathrm{UV} \lambda_{\max }($ metanol $): 216 \mathrm{~nm}(\log \varepsilon=4.38), 232 \mathrm{~nm}(\log \varepsilon=$ 4.35), $276 \mathrm{~nm}(\log \varepsilon=4.28), 314 \mathrm{~nm}(\log \varepsilon=3.90)$. IR. $(\mu): \mathrm{F}_{2}$ de izlenen $-\mathrm{OH}$ bandının kaybı yanısıra, 3.44 - 3.53 (alkil -CH), 5.92 (4. konum $\mathrm{C}=\mathrm{O}$ ), 5.75 (eter $\mathrm{C}=\mathrm{O}$ ), 7.84, 8.33 (fenil-alkil eter gerilme), 8.08, 8.55 (C-CO-O deformasyon), 9.43 (O-C-C deformasyon). NMR. (İnt. TMS, $\mathrm{CDCl}_{3}, \delta \mathrm{ppm}$ ) : 8.2 (d. $\mathrm{H}-5$ ), 7.7 (d. 2' 6' - protonlar1), 7.2 (d. 3', 5' - protonlar1), 6.9 (q. H-6), 6.7 (d. H8), 5.6 (d. d. H-2), 4.0 (s. 4' - $\mathrm{OCH}_{3}$ ), 2.9 - 3.09 (d. d. H-3). Eter yan zinciri : 4.9 (s. $-\mathrm{O}-\mathrm{CH}_{2}-$ ), 4.4 (q. etil esteri $-\mathrm{CH}_{2}-$ ), 1.3 (t. etil esteri $\left.-\mathrm{CH}_{3}\right)$. Mass : $\mathrm{m} / \mathrm{e}=356\left(\mathrm{M}^{+}\right), 269,249,161,134,121$, 119, 108, 107, 91. Anal. : $\mathrm{C}_{2} \mathrm{OH}_{20} \mathrm{O}_{6}$ için Hesaplanan : $\mathrm{C}: 67.41 ; \mathrm{H}$ : 5.61, Bulunan : C : 67.46; H : 5.87 .

114 - Okso - 2 - (4' - metoksi - fenil) - 2,3 - dihidrobenzopiran 7 - ili oksil - $\alpha$ - metil asetik asit etil esteri; 7 - (4' - meoksi - flavanon) oksi $-\alpha$ - metil asetik asit etil esteri $=F_{2} P: 0.405 \mathrm{~g}(0.0015$ mol) $\mathrm{F}_{2}$ üzerine $0.271 \mathrm{~g}(0.0015 \mathrm{~mol}) \alpha$ - bromopropiyonik asit etil esteri, $0.207 \mathrm{~g}$ ( $0.0015 \mathrm{~mol})$ susuz $\mathrm{K}_{2} \mathrm{CO}_{3}$ ve $25 \mathrm{ml}$ kuru aseton ilave edildi. $\mathrm{F}_{2} \mathrm{~A}$ 'da verilen işlemler aynen tekrarlanarak hiç bir çözücüde kristallenmeyen açık sarı renkli, koyu kıvamlı sıvı bir madde elde edildi. Verim \% 72,1, Eter, aseton, $\mathrm{MeOH}, \mathrm{EtOH}, \mathrm{CHCl}_{3}$ ve petrol eterinde çözünür. \% $5 \mathrm{FeCl}_{3}$ ile renksiz, Shinoda testi ile koyu mavi - menekşe renk reaksiyonu verir. $\mathrm{Rf}=0.24$ (solvan A), 0.69 (solvan $\mathrm{C}$ ).

UV $\lambda_{\max }($ metanol $): 216 \mathrm{~nm}(\log \varepsilon=4.43), 232 \mathrm{~nm}(\log \varepsilon=$ 4.3S), $276 \mathrm{~nm}(\log \varepsilon=4.30), 314 \mathrm{~nm}(\log \varepsilon=3.92)$. IR. $(\mu)$ : 3.34 - 3.53 (alkil $\mathrm{C}-\mathrm{H}), 5.67$ (ester $\mathrm{C}=\mathrm{O}), 5.92$ (4. konum $\mathrm{C}=\mathrm{O})$, 7.94 (fenil - alkil eter gerilme), 8.13, 8.55 (C-CO-O), 9.7 (O-C$\mathrm{C}$ deformasyon). NMR (İnt. TMS, $\mathrm{CDCl}_{3}, \delta \mathrm{ppm}$ ) : 8.2 (d. H-5), 7.8 (d. 2', 6' - protonlar1), 7.3 (d. 3', 5' - protonlar1), 6 (q. H-6), 6.8 (d. $\mathrm{H}-8$ ), 5.8 (d. d. $\mathrm{H}-2$ ), 4.0 (s. 4 ' $-\mathrm{OCH}_{3}$ ), 3.0 - 3.1 (d. d. H-3); eter yan zinciri : 5.0 (q. etere komşu -CH-) 4.4 (q. etil ester grubu $-\mathrm{CH}_{2}-$ ), 1.78 (d. etere komşu karbon'un taşıdığ $1 \mathrm{CH}_{3}$ ), 1.3 (t. etil ester grubu $\left.-\mathrm{CH}_{3}\right)$. Mass. $\mathrm{m} / \mathrm{e}=370\left(\mathrm{M}^{+}\right)$, 297, 269, 262, 168, 161, 134, 121, 108, 91, 77, Anal. : $\mathrm{C}_{21} \mathrm{H}_{22} \mathrm{O}_{6}$ için, Hesaplanan : $\mathrm{C}$ : 68.10; H : 5.94, Bulunan : C : 68.17; H : 5.73.

165 


\section{Rahmiye ERTAN}

114 - Okso - 2 - (4' - metoksi - fenil) - 2,3 - dihidrobenzopiran 7 - ill oksil - $\alpha$ - etil - asetik asit etil esteri; 7 - (4' - metoksi - flavanon) oksi $-\alpha$ - etil asetik asit etil esteri $=\mathrm{F}_{2} \mathrm{~B}: 0.405 \mathrm{~g}(0.0015$ mol) $\mathrm{F}_{2}$ üzerine $0.293 \mathrm{~g}(0.0015 \mathrm{~mol}) \alpha$ - bromobütirik asit etil esteri, $0.207 \mathrm{~g}(0.0015 \mathrm{~mol})$ susuz $\mathrm{K}_{2} \mathrm{CO}_{3}$ ve $25 \mathrm{ml}$ kuru aseton ilave edildi. $\mathrm{F}_{2} \mathrm{~A}^{\prime}$ da verilen şartlarda 12 saat kaynatıld 1 ve diğer işlemler aynen tekrarland1. Hiç bir çözücüde kristallenmeyen açık sarı renkli, koyu kıvamlı sıvı bir madde elde edildi, verim \% 71.12, eter, aseton, $\mathrm{MeOH}, \mathrm{EtOH}, \mathrm{CHCl}_{3}$ ve petrol eterinde çözünür. \% $5 \mathrm{FeCl}_{3}$ ile renksiz, Shinoda testi ile mavi - menekşe renk reaksiyonu verir. $\mathrm{Rf}=$ 0.26 (solvan A), 0.70 (solvan $\mathrm{C}$ ).

$\mathrm{UV} \lambda_{\max }($ metanol $): 216 \mathrm{~nm}(\log \varepsilon=4.39), 3,22 \mathrm{~nm}(\log \varepsilon=$ 4.35), $276 \mathrm{~nm}(\log \varepsilon=4.28), 314 \mathrm{~nm}(\log \varepsilon=3.90)$. ER. $(\mu): 3.34-$ 3.52 (alkil -CH-), 5.68 (ester $\mathrm{C}=\mathrm{O}), 5.91$ (4. konum halka $\mathrm{C}=0$ ), 7.93 (fenil-alkil eter gerilme), 8,13 - 8.54 (C-CO-O ester deformasyon), 9.70 (O-C-C deformasyon). NMR. (Int. TMS, $\mathrm{CDCl}_{3}, \delta \mathrm{ppm}$ ) : 8.2 (d. H-5), 7.7 (d. 2', 6' - protonlar1), 7.2 (d. 3', 5' - protonlar1), 6.9 (q. H - 6), 6.8 (d. H - 8), 5.6 (d. d. H - 2), 4.0 (s. 4' - $\mathrm{OCH}_{3}$ ), 2,9 3.1 (d. d. H-3). Eter yan zinciri : 4,7 (t. etere komşu -CH-), 4.4 (q. etil ester grubu - $\mathrm{CH}_{2}-$ ), 2.0 (m. $\alpha$ - etil grubu $\mathrm{CH}_{2}-$ ), $0.9-1.2$ (t. $6 \mathrm{H}$, etil ester grubu $-\mathrm{CH}_{3}$ ve $\alpha$ - etil grubu $-\mathrm{CH}_{3}$ ). Mass : $\mathrm{m} / \mathrm{e}=$ $384\left(\mathrm{M}^{+}\right), 311,277,269,177,161,134,119,108,91,77$. Anal. : $\mathrm{C}_{22} \mathrm{H}_{24} \mathrm{O}_{6}$ için Hesaplanan : $\mathrm{C}: 68.75 ; \mathrm{H}: 6.25$, Bulunan : $\mathrm{C}: 68.63$; $\mathrm{H}: 6.07$.

114- Okso - 2 - (4' - metoksi - fenil) - 2, 3 - dihidrobenzopiran 7 - ill oksil - $\alpha$ - propilasetik asit etil esteri; 7 - (4' - metoksi - flavanon) oksi $-\alpha$ - propilasetik asit etil esteri $=\mathrm{F}_{2} \mathrm{~V}: 0.405 \mathrm{~g}$ $(0.0015 \mathrm{~mol}) \quad \mathrm{F}_{2}$ üzerine $0.315 \mathrm{~g}(0.0015 \mathrm{~mol}) \alpha$ - bromovalerianik asit etil esteri, $0.207 \mathrm{~g}(0.0015 \mathrm{~mol})$ susuz $\mathrm{K}_{2} \mathrm{CO}_{3}$ ve $25 \mathrm{ml}$ kuru aseton ilave edildi. $\mathrm{F}_{2} \mathrm{~A}^{\prime} \mathrm{da}$ verilen şartlarda 12 saat kaynatıldı, ve diğer temizleme işlemleri aynen tekrarland1. Hiç bir çözücüde kristallenmeyen sarımsı beyaz renkli, koyu kıvamlı siv1 bir madde elde edildi, verim \% 70.4. Eter, aseton, $\mathrm{EtOH}, \mathrm{MeOH}, \mathrm{CHCl}_{3}$ ve petrol eterinde çözünür. \% $5 \mathrm{FeCl}_{3}$ ile renksiz, Shinoda testi ile mor - menekşe renk reaksiyonu verir. $\mathrm{Rf}=0.30$ (solvan $\mathrm{A}), 0.70$ (solvan $\mathrm{C}$ ).

UV $\lambda_{\max }($ metanol) : $216 \mathrm{~nm}(\log \varepsilon=4.39), 282 \mathrm{~nm}(\log \varepsilon=$ 4.30), $276 \mathrm{~nm}(\log \varepsilon=4.28), 314 \mathrm{~nm}(\log \varepsilon=3.93)$. IR. $(\mu)$ : 


\section{Spazmolitik Etki Gösteren Bazı Flavanon Eter Türevleri}

3.38 - 3.53 (alkil -CH-), $5.68($ ester $\mathrm{C}=\mathrm{O}), 5.92(4$. konum $\mathrm{C}=\mathrm{O})$. 7.94 (fenil-alkil eter gerilme), 8.13 - 8.62 (C-CO-0 ester deformasyon), 9.71 (O-C-C deformasyon). NMR. (İnt. TMS, $\left.\mathrm{CDCl}_{3}, \delta \mathrm{ppm}\right)$ : 8.2 (d. H-5), 7.8 (d. 2', 6'-'protonlar1), 7.2 (d. 3', 5'-protonlar1), 6.9 (q. H-6), 6.7 (d. H-8), 5.7 (d. d. H-2), 4.0 (s. 4' - $\mathrm{OCH}_{3}$ ), 2.95 3.1 (d. d. H-3), Eter yan zinciri : 4.8 (t. etere komşu -CH), 4.4 (q. etil esteri-CH2-), $1.6-2.0$ (m. $4 \mathrm{H}$ integral değeri ile valerianik aside ait metilen protonlar 1$), 0.9-1.3$ (6H integral değeri ile etil esteri $\mathbf{- C H 3}$ ve valerianik aside ait $-\mathrm{CH}_{3}$ protonlar1). Mass. : $\mathrm{m} / \mathrm{e}=398$. W) + ), 325, 291, 269, 191, 161, 134, 121,119, 108, 91. Anal. : $\mathrm{C}_{23} \mathrm{H}_{26} \mathrm{O}_{6}$ için; Hesaplanan : $\mathrm{C}: 69.34 ; \mathrm{H}: 6.53$, Bulunan : $\mathrm{C}: 69.28$; $\mathrm{H}: 6.41$.

[[4 - Okso - 2 - (4' - metoksi - fenil) - 2,3 - dihidrobenzopiran 7 - ill oksil - $\alpha$ - fenilasetik asit etil esteri; 7 - (4' - metoksi-flavanon) oksi $-\alpha-$ fenil-asetik asit etil esteri $=\mathrm{F}_{2} \mathrm{~F}$ : $0.405 \mathrm{~g}(0.0015$ mol) $\mathrm{F}_{2}$ üzerine, $0.365 \mathrm{~g}(0.0015 \mathrm{~mol}) \alpha$ - bromofenil asetik asit etil esteri, $0.207 \mathrm{~g}(0.0015 \mathrm{~mol})$ susuz $\mathrm{K}_{2} \mathrm{CO}_{3}$ ve $25 \mathrm{ml}$ kuru aseton ilave edildi. $\mathrm{F}_{2} \mathrm{~A}^{\prime} \mathrm{da}$ verilen şartlarda 14 saat kaynatıldı, ve diğer temizleme işlemleri aynen tekrarlandı. Etanol-aseton karışımından elde edilen beyaz renkli kristallerin En. $=92-95^{\circ} \mathrm{C}$, verim \% 66.4 Eter, asetonda çok çözünür. EtOH, $\mathrm{MeOH}, \mathrm{CHCI}_{3}$ da çözünür. \% $6 \mathrm{FeC}_{3}$ ile renksiz, Shinoda testi ile vişne çürüğ̈ renk reaksiyonu verir, $\mathrm{Rf}=0.24$ (solvan A), 0.66 (solvan C).

$\mathrm{UV} \lambda_{\max }($ metanol $): 220 \mathrm{~nm}(\log \varepsilon=4.52), 276 \mathrm{~nm}(\log \varepsilon=$ 4.33), $314 \mathrm{~nm}(\log \varepsilon=3.93)$. IR. $(\mu): 3.44-3.53$ (alkil -CH-), 5.65 $($ ester $\mathrm{C}=\mathrm{O}$ ), 5.92 (4. konum $\mathrm{C}=\mathrm{O}), 7.87$ (fenil-alkil eter gerilme), 8.13 - 8.62 (C-CO-0 ester deformasyon), 9.17 (O-C-C deformasyon). NMR. (İnt. TMS, $\left.\mathrm{CDC}_{3}, \delta \mathrm{ppm}\right): 8.2$ (d. $\left.\mathrm{H}-5\right), 7.8(7 \mathrm{H}$ integral değeri ile 2', 6' - protonları ve eter yan zincirindeki fenil halkas1 protonlar1), 7.2 (d. 3', 5'- protonlar1), 7.0 (q. H-6), 6.8 (d. H-8), 5.8 (d. d. H-2), 4.0 (s. 4' $-\mathrm{OCH}_{3}$ ), 2.9-3.1 (d. d. H-3). Eter yan zinciri : 5.8 (s. etere komşu -CH-), 4.4 (q. ester etil grubu . $\mathrm{CH}_{2}-$ ), 1.2 (t. ester etil grubu $\left.-\mathrm{CH}_{3}\right)$. Mass. : $\mathrm{m} / \mathrm{e}=432(\mathrm{M}+$ ), $388,359,289,225,163,135,134,119,107,91$. Anal. : $\mathrm{C}_{26} \mathrm{H}_{24}, 0_{6}$ için; Hesaplanan : $\mathrm{C}: 72.22 ; \mathrm{H}: 5.95$, Bulunan : $\mathrm{C}: 71.97 ; \mathrm{H}$ : 5.86 . 
7 - Metoksi - 4 - okso - 2 (4' - metoksifenil) - 2,3 - dihidrobenzopiran; 7,4' - Dimetoksi-flavanon $=\mathbf{F}_{2} \mathbf{M}: 0.405 \mathrm{~g}(0.0015 \mathrm{~mol})$ $\mathbf{F}_{2}$ üzerine $0.355 \mathrm{~g}(0.0025 \mathrm{~mol})$ metil iyodür, $0.207 \mathrm{~g}(0.0015 \mathrm{~mol})$ susuz $\mathrm{K}_{2} \mathrm{CO}_{3}$ ve $25 \mathrm{ml}$ kuru aseton ilave edildi, $\mathbf{F}_{2} \mathbf{A}^{\prime}$ 'da verilen şartlarda 8 saat kaynatıld1 ve $\mathrm{F}_{2} \mathrm{~A}^{\prime}$ daki temizleme işlemleri aynen uyguland1. Aseton-eter karışımından kristallendirilen maddenin En. = $92-94^{\circ}\left(94-95^{\circ} \mathrm{C}\right.$ Lit. (9)), verim \% 70.4. Eter, asetonda çok çözünür, $\mathrm{EtOH}, \mathrm{MeOH}, \mathrm{CHCl}_{3}$ da çözünür. \% $5 \mathrm{FeCl}_{3}$ ile renksiz, Shinoda testi ile koyu - k1rmızı renk reaksiyonu verir. $\mathrm{Rf}=0.36$ (solvan A), 0.67 (solvan C), 0.82 (solvan D).

$\mathrm{UV} \lambda_{\max }($ metanol $): 216 \mathrm{~nm}(\log 3=4.25), 232 \mathrm{~nm}(\log \varepsilon=$ 4.25), $275 \mathrm{~nm}(\log \varepsilon=4.26), 314 \mathrm{~nm} \quad(\log \varepsilon=3.85)$. IR. $(\mu)$ : 3.38 - 3.52 (alkil -CH-), 5.95 (4. konum $\mathrm{C}=0$ ), 7.87 (fenil-alkil asimetrik eter gerilme), 9.80 (eter grubu deformasyon). NMR. (İnt. TMS, $\left.\mathrm{CDCl}_{3}, \delta \mathrm{ppm}\right): 8.2(\mathrm{~d} . \mathrm{H}-5), 7.8 \mathrm{~d}\left(2^{\prime}, 66^{\prime}\right.$ - protonlar1), 7.2 (d. 3', 5' - protonlar1), 6.9 (q. H-6), 6.7 (d. H-8), 5.7 (d. d. H-2), 4.0 (s. $6 \mathrm{H}$ integral değeri ile 4 ' ve 7 -metoksi grubu protonlar1), $3.0-3.1$ (d. d. H-3). Mass. : $\mathrm{m} / \mathrm{e}=284\left(\mathrm{M}^{+}\right), 270,177,150,135$, 134, 122, 121, 119, 107, 91. Anal. : $\mathrm{C}_{17} \mathrm{H}_{16} \mathrm{O}_{4}$ için; Hesaplanan : $\mathrm{C}$ : 71.83; H : 5.63; Bulunan : $\mathrm{C}: 71.67, \mathrm{H}: 5.52$.

\section{SONUÇ ve TARTIŞMA}

7 - hidroksi - 4' - metoksi - flavanon $\left(\mathrm{F}_{2}\right)$ halkası sentez edildikten sonra, halka üzerindeki serbest $7-\mathrm{OH}$ grubu üzerinden çeşitli a - bromo - a - sübstitue asetik asit etil esterleri ile eterleştirilmesi sağlanarak :

$\alpha$ - bromo-asetik asit etil esteri ile

$\alpha$-bromo- $\alpha$-metil asetik asit etil esteri ile

$\alpha$ - bromo $-\alpha$ - etil asetik asit etil esteri ile

$\alpha$-bromo $-\alpha$-propil asetik asit etil esteri ile

$\alpha$ - bromo $-\alpha$ - fenil asetik asit etil esteri ile

Aynı halkanın 'metil iyodür' ile

$\left(\mathrm{F}_{2} \mathrm{M}\right)$ türevleri elde edilmiştir. Elde edilen bu eter türevlerinin, literatürde verilen patent yöntemi üe (1) sentezlenen "Efloxate" bileşiği referans alınarak $\mathrm{BaCl}_{2}$, asetil kolin ve histamin agonistlerine karşı spazmolitik etkileri incelenmiştir. Bundan önceki çalışmada olduğu gibi buradaki uygulamada da aynı yöntemden (10) yararlanılmış- 


\section{Spazmolitik Etki Gösteren Bazı Flavanon Eter Türevleri}

tır. Buna göre incelemeler cinsel fark gözetmeksizin $400-500 \mathrm{~g}$ ağırlığındaki kobaylardan ahnan terminal ileum parçaları üzerinde ve izole organ banyosunda, sentez edilen maddelerin $1 \times 10-^{5} \mathrm{M} / \mathrm{ml}$ konsantrasyonları ile yapılmıştır. \% İnhibisyon değerlerinin saptanabilmesi için önce agonist maddelerin (asetil kolin, histamin, $\mathrm{BaCl}_{2}$ ) \% maksimum kasılmaları kaydedilmiş, daha sonra antagonistlere (sentez edilen türevlere) karşı cevaplan ölçülmüştür. Kas1lmalar, palmer isli tambura veya 7005 - UGO Basil transducer aracılığı ile 7050 - UGO Basil mikrodinamometreye kaydedilmiştir. Her agonist için en az üç aynı kasılma boyunu veren cevaplar standart olarak alınmıştır. Asetil kolin ve histamin için tekrarlanan dozlar arasındaki bekleme süresi 2 dakika. $\mathrm{BaCl}_{2}$ için 10 dakika olarak alınmıştır. Antagonist etkinin saptanmasında incelenen maddeler ileum ile 30 dakika inkübasyona bırakılmıştır. Antagonistlere karşı bütün agonistler için yeniden kaydedilen cevaplardaki doz aralığ 2 dakikaya indirilmiştir. Böylece agonistlerle elde edilen kasılma boyu ile antagonistler verildikten sonra elde edilen kasılma boyu oranlanarak \% spazmolitik etkiler saptanmıştır. Her türev için en az iki defa tekrarlanan deney sonuçları Student " $t$ " testine göre istatistiksel olarak değerlendirilmiştir. Ancak deney sayısının az olduğu türevler için güven aralığı (güvenirlik sınırları) doğal olarak geniş çıkmıştır. Elde edilen \% inhibisyon değerleri Tablo : 1 de bir arada verilmiştir.

Tablo değerleri incelendiği zaman, halkanın bizzat kendisinin de eter köprüsü ile bağlı ester yan zincirli türevler yanı sıra bir miktar spazmolitik etki içerdiği görülmektedir. Elde edilen türevlerin asetil koline olan etkileri Efloxate'tan daha düşük olmakla birlikte $\mathrm{BaCl}_{2}$ ve histamine karşı daha yüksek etkili türevler de mevcuttur $\left(\mathrm{BaCl}_{2}\right.$ e karş1 $\mathbf{F}_{2} \mathbf{P}$ ve $\mathbf{F}_{2} \mathbf{B}$; histamine karşı $\mathbf{F}_{2} \mathbf{V}$ ve $\left.\mathbf{F}_{2} \mathbf{F}\right)$. Ayrıca $\mathbf{F}_{2} \mathbf{P}$ ve $\mathbf{F}_{2} \mathbf{B}$ her üç agoniste karşı da \% 80'in üstünde bir inhibisyon gösterdiklerinden daha detaylı bir farmakolojik incelemeye değer görülebilir. $\mathbf{F}_{2} \mathbf{M}$ ile diğer $\mathbf{F}_{2}$ türevleri mukayese edildiği zaman, tablo değerlerinden de görüldüğü gibi $\mathbf{F}_{2} \mathbf{M}^{\prime}$ 'in daha az etkili olduğu söylenebilir. Böylece bu türevler için, eter-ester yan zincirinin aktivite üzerinde rol oynadığı düşünülebilir.

Elde edilen $\mathrm{F}_{2}$ türevlerinin de, bundan önceki çalışmada verilen (2) $\mathbf{F}_{1}$ türevlerinde olduğu gibi, farmakolojik deneyler sırasın- 
Tablo I. (Farmakolojik Etki Sonuçları)

\begin{tabular}{|c|c|c|c|c|c|c|c|c|c|c|c|c|c|c|c|c|c|c|c|c|}
\hline \multirow{2}{*}{$\begin{array}{c}\text { Agonist } \\
\text { Antagonist } \\
1 \times 10.5 \mathrm{M} / \mathrm{ml}\end{array}$} & \multicolumn{20}{|c|}{$\% \quad \dot{I} \mathbf{N} \quad \mathbf{H}$ İ $\mathbf{B}$ İ $\mathbf{S}$ Y O $\mathbf{N}$} \\
\hline & \multicolumn{6}{|c|}{$\begin{array}{l}\text { Asetil kolin } \\
\left(\begin{array}{llll}6.4 & \times & 10-7 & \mathrm{M} / \mathrm{ml}\end{array}\right)\end{array}$} & \multicolumn{7}{|c|}{\begin{tabular}{c}
\multicolumn{2}{c}{ Histamin } \\
$\left(\begin{array}{llll}1 & \mathbf{x} & \mathbf{1 0}-8 & \mathrm{M} / \mathrm{ml}\end{array}\right)$
\end{tabular}} & \multicolumn{7}{|c|}{\begin{tabular}{llll} 
& \multicolumn{4}{c}{$\mathbf{B a C l}_{2}$} \\
$(5$ & $\mathbf{x}$ & $10-3$ & $\mathbf{M}$
\end{tabular}} \\
\hline $\mathbf{F}_{2}$ & 77.81 & & \pm 0.82 & $(75.4$ & $\sim \mathbf{8 0 . 1})_{3}$ & (4) 4 & 55.7 & \pm & 2.8 & $(47.0$ & $\sim$ & $64.5)$ & (4) & 74.1 & \pm & 0.8 & (71.8 & $\sim$ & 76.6) & (4) \\
\hline $\mathbf{F}_{2} \mathbf{A}$ & 89.9 & \pm & 1.3 & $(85,9$ & 93.9) & (4) & 80.6 & + & 1.3 & $(76.6$ & $\sim$ & 84.6) & (4) & 60.3 & \pm & 0.8 & $(57.9$ & $\sim$ & 62.7) & (4) \\
\hline $\mathbf{F}_{2} \mathbf{P}$ & 83.3 & \pm & 2.7 & $(76.5$ & $\sim 90.2)$ & (6) & 86.2 & \pm & 1.8 & $(80.7$ & $\sim$ & $91.8)$ & (4) & 87.0 & \pm & 3.8 & $(76.6$ & $\sim$ & $97.5)$ & (5) \\
\hline $\mathbf{F}_{2} \mathbf{B}$ & 88.7 & \pm & 1.3 & $(84.8$ & $\sim 92.7)$ & (4) & 84.5 & \pm & 3.3 & $(74.1$ & $\sim$ & 94.8) & (4) & 95.4 & \pm & 1.8 & $(89.9$ & $\sim$ & $100.9)$ & (4) \\
\hline $\mathbf{F}_{2} \mathbf{V}$ & 91.0 & \pm & 1.3 & (87.0 & $\sim 95.0)$ & (4) & 95.3 & \pm & 1.0 & $(82.5$ & $\sim$ & $108.1)$ & (2) & 52.3 & \pm & 3.5 & ( 7.5 & $\sim$ & 97.1) & (2) \\
\hline $\mathbf{F}_{2} \mathbf{F}$ & 88.1 & \pm & 1.0 & (85.9) & $\sim 91.0)$ & (6) & 94.1 & \pm 0 & & $(91.8 \sim$ & & $96,5)$ & (4) & 47.5 & \pm & 4.2 & (34.0 & $\sim$ & $61.0)$ & (4) \\
\hline $\mathbf{F}_{2} \mathbf{M}$ & 52.7 & \pm & 2.5 & $(20.7$ & $\sim$ 84.7) & (2) & 57.3 & \pm & 2.5 & $(25.3$ & & $89.3)$ & (2) & 50.4 & \pm & 3.0 & $(12,0$ & $\sim$ & 88.8) & (2) \\
\hline Efloxate & 93.4 & \pm & 3.3 & (83.1 & $\sim 103.8)$ & (4) & 86.3 & \pm & 2.3 & $(79.2$ & $\sim$ & 93.5) & (4) & 76.3 & \pm & 4.3 & $(62.8$ & $\sim$ & 89.8) & (4) \\
\hline
\end{tabular}

$1-\overline{\mathrm{x}}$ (aritmetik ortalama)

2 - S. E. M. $=\frac{\mathrm{S}}{\sqrt{\mathrm{n}}}$ (aritmetik ortalamanın standart hatası; $\mathrm{S}=$ standart sapma, $\mathrm{n}=$ deney sayısı)

3 - Güvenirlik sınırları - t x (S. E. M.); t = n-1 serbestlik derecesi için \% 95 olasılıklı tablo değeri M $4-\mathbf{n}=$ Deney sayısı

170 


\section{Spazmolitik Etki Gösteren Bazı Flavanon Eter Türevleri}

da düz kaslar üzerine tersinir (reversibl) bir etki içerdikleri gözlenmiştir. Bu da bu maddeler için oldukça iyi bir özelliktir.

\section{ÖZET}

Bu çalışmada 7 - hidroksi - 4' - metoksi - flavanon halkası sentez edilmiş ve sonra bu bileşiğin serbest hidroksil grubu üzerinden, çeşitli $\alpha$ - bromo - $\alpha$-substitue asetik asit etil esterleri ile eterleştirme reaksiyonları incelenmiştir. Daha sonra Efloxate (Recordil) adı verilen bileşik referans alınarak, elde edilen eter türevlerinin asetil kolin, histamin ve $\mathrm{BaCl}_{2}$ agonistlerine karşı spazmolitik etkileri araştırılmıştır.

\section{RESUME}

Dans ce travail nous avons synthétisé le 7 - hydroxy - 4' - methoxy - flavanone et ensuite examiné les réactions d'étherification sur le groupe de 1' -OH libre de la flavanone obtenue, avec certain ethyl ester de l'acide acétique $\alpha$ - bromo - $\alpha$ - substitué. Puis, en prenant l'Efloxate (Recordil) comme référence, nous avons étudié l'activité spasmolytique de tous ces dérivés vis-a-vis des agonistes tels que l'acétylcholine, l'histamine et le $\mathrm{BaCl}_{2}$.

\section{TEŞEKKÜR}

$\mathrm{Bu}$ çalışmadaki farmakolojik deneylerin yapılmasında, fakültemiz Farmakoloji Kürsüsü Başkanı Sayın Prof. Dr. Orhan Altınkurt ve asistanlarına değerli yardımlarından dolayı teşekkürü bir borç bilirim.

\section{LITERATÜR}

1. Colleoni, D. R., Setnicar D. R., Brit. P. 803, 327, 824, 547 (1958 and 1959 to Dr. Recordati - Daboratorio Farmacol. S.P.A.)

2. Ertan, R., A. Ü. F,Ecz. Fak. Mec, 11, 9 - 22 (1981)

3. Shinoda, J., J. Pharm. Soc. Japan., 48, 214 (1928); Ref. C. A. 22, 2947 (1928)

4. Brieskorn, C. H. und MEISTER, G., Arch. Pharm. 298, 435 (1965)

5. Wasserman, H. H. and Aubrey, N. E., J. Amer. Chem. Soc. 77, 590 (1955)

6. Pineau, J-P. et Chopin, 3., Pull. Soc. Chim. France., 3678 (1971)

7. Aubry, C. et Chopin, J., Ibid, 4503 (1971)

8. Geissman, T. A. and Clinton, O. R., 3. Amer. Csem. Soc. 68, 697 (1946)

9. Juppen, K. and Kostanecki, St. V., Chem. Ber. 37, 4160 (1904)

10. Turner, R. A. Screening Methods in Pharmacology. Vol. I. Academic Press New York London (1965) 